\section{MIES: CONCURSOS EN LA FRIEDRICHSTRASSE}

MIES: COMPETITIONS IN FRIEDRICHSTRASSE

Cristina Gastón Guirao

\section{Vista de la Friedrichstrasse desde}

$E$

ntre los años 1910 y 1934 Mies participó en una (docena de competiciones en Alemania: siele el área urbana más centrica y comercial de cuatro de ens area de radio menor a un quilomerrocuatro de ellas sobre la misma calle Friedrichstrasse, incluso, en dos ocasiones, sobre el mismo emplazamiento. en el Werkbund ni desarrollar (figura 1).

Siendo muy joven había participado en dos concursos importantes: el del Monumento a Bismarck (1910) - con tan sôlo 24 años-y la competición encubierta para la residencia de los Kroller-Muller en Wassenaar (1912) en los que despunto. Ahora bien, es su priner concurso en Berlin, para un rascacielos jutito a la estación Friedrichstrasse (1921/22), el que le situa por primera vez ante el compromiso que supone intervenir en la ciudad consolidada y le hace ganar un inaudio prestiglo profesional. Mies obluvo menciones honolicas en varios concursos pero nunca un primer premio. Al trabajar, sus inquietudes siempre excedian la cuestión particular planteada se afanaba por la arquitectura más que por satisfacer al promotor. Ello tenía consecuenclas como el presentar

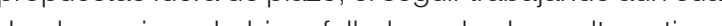

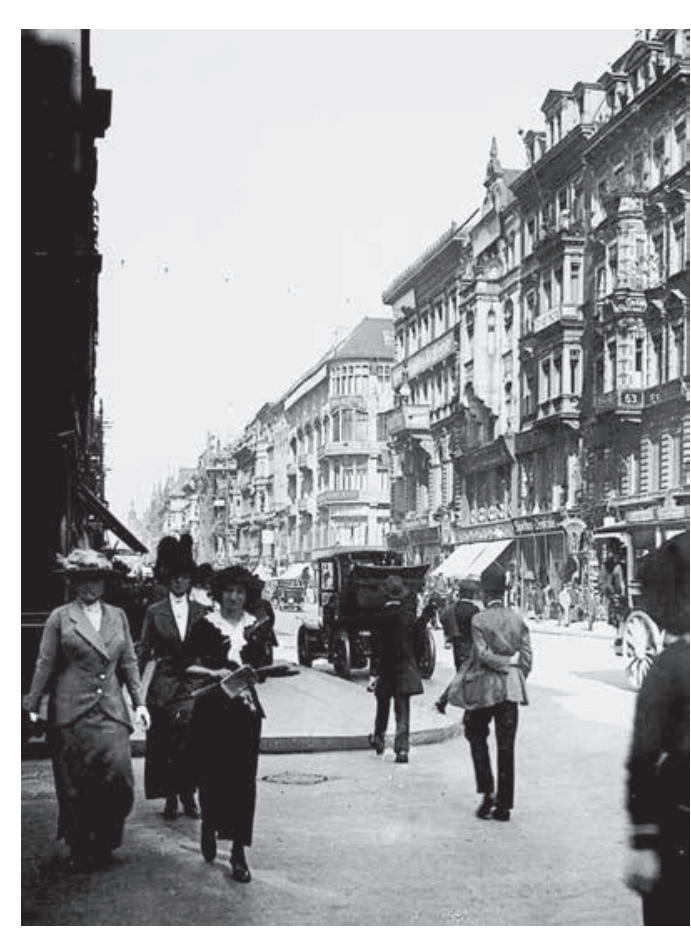

una localización pasados varios años. En el año 1928 almacenes comerciales S. Adam, en la esquina de la Frie drichstrasse con la Leipzigerstrasse, encadena la partcipacion en una serie concursos a los que se le invitara expresamente.

Los proyectos presentados a concurso ofrecen oporbrindan la posibilidad de ver diferentes maneras de resolver una misma ouestion. Pero además, en los concursos que nos ocupan se da la circunstancia añadida de la coincidencia de sus localizaciones. El ejercicio, con cas una década de direrencla sobre la misma parcela, permite apreciar qué convicciones maniene el autor o cuales han cambiado, asi como medir su evolución respecto de la de sus contemporaneos.

Las propuestas de Mies se distinguen tanto por e planteamiento arquitectónico como por el modo de presentarks. Las composiciones gánicas que elaboraba revelan una mirada minuciosa al mundo. A pesar de la autonomia que exhiben sus actuaciones, Mies siempre siluaba su intervención con relación a lo que sucedia alEl sigu desdo una conciencia excepcionalmente lúcida. 年 


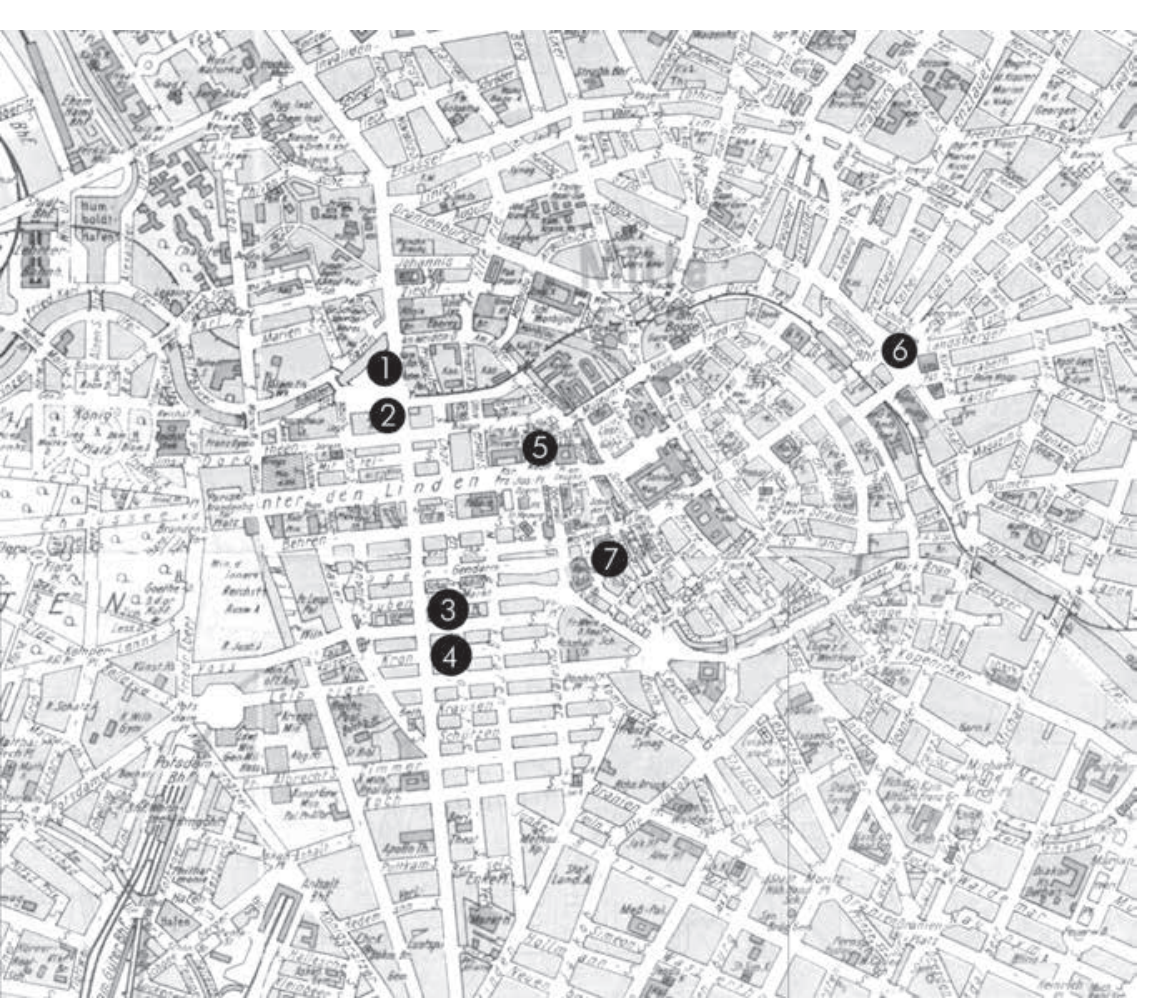

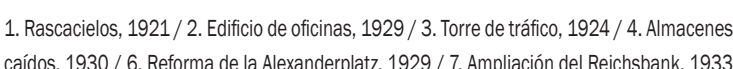

que generó en tanto que informan de un modo de encarar la relación entre el edificio y la ciudad. La autora encontró el hilo para este artículo preparando el plano de ubicación de los concursos de Mies en Berlin (figura 2).

tuvo un gran éxito pese a lo exiguo del plazo: se presentaron 145 propuestas. La de Mies fue descalificada: contravenia las bases en numerosos puntos y difería en mucho del tono de las demas propuestas, tanto en las especificaciones como en el modo de presentación. No se incluyó ni entre las publicadas en el folleto editado para dar cuenta de los resultados

1921/22. JUNTO A LA ESTACIÓN FRIEDRICHSTRASSE

La parcela

El emplazamiento del concurso se situaba en una zona centrica comercial muy transitada, saturada de hoteles y locales de ocio: una parcela triangular que ya había sido objeto de viene detenerse en las particulares circun localizacion, su geometria y las caracteristicas de los frenes edificados que la rodeaban. Las bases altura para amoliguar el impacto sobre las edilicaciones aledanas e imponian condiciones especificas en base a ciertos estudios previos realizados

el proyecto de un rascacielos destapó sus anhelos. La fecha limite de recepción de propuestas se

1. En 1988 , el Bauhaus-Archiv y la Technische Universität Berlin organizan una exposición y editan una publicación sobre el concuurso para el rascacielos de 1. una selección de 40 proyectos presentados. Constituye la mayor base documental sobre este concurso y ha sidd referencia fundamental para este articulo. 2. Vittorio M. Lampugnani hace una exhaustiva explicación de la actividad urbanística y arquitectónica en el Berlín de las primeras décadas del siglo XX en el

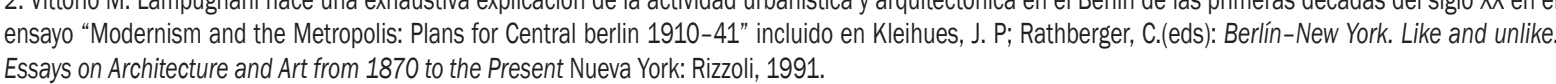
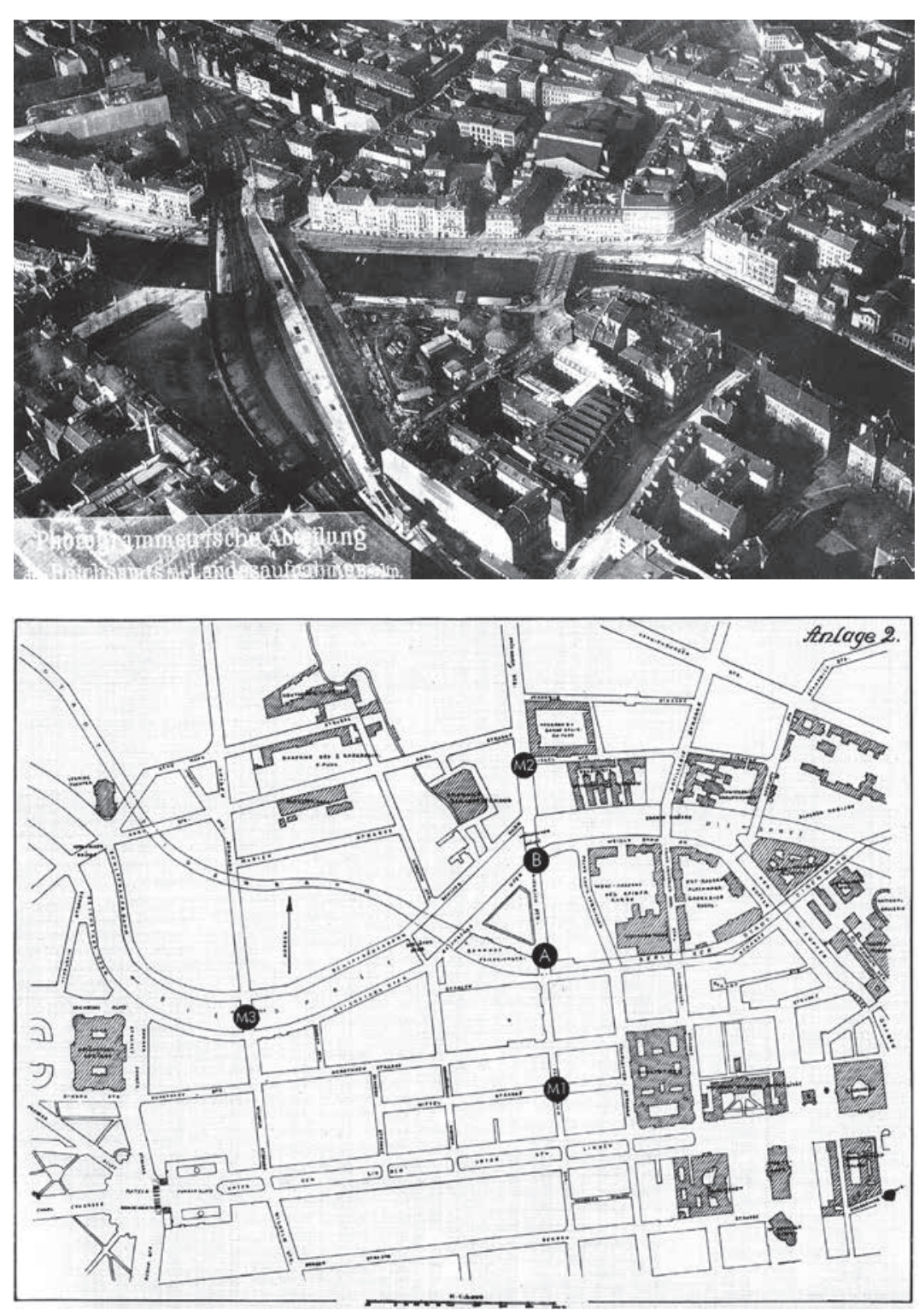

La organización facilitó un plano de situación a escala 1:400, uno de la planta del solar a escala 1:200 y dos vistas aereas, desde el sureste y desde el suroeste. El solar rriangular contaba aproximadamente con $4800 \mathrm{~m}^{2}$. Las longitudes de los lados eran $100^{\prime} 5$ metros en dirección al rio Spree, 95 metros hacia la estacióny 89 metros a la calle Friedrichstrasse -con dos chaflanes de 15 metros hacia el puente y de 3 metros hacia el paso elevado-. La acera opuesta de la Friedrichstrasse estaba ocupada por un frente continuo de edificios de planta baja y tres pisos entre los que estaban el Hotel Monopol y la Oper Komisher. Las vias cruzaban la Friedrichstrasse por un paso elevado y el edificio de la estación consistía en una gran estructura metalica, sobre muros de obra vista de color producía desde el lateral opuesto. El tercer lado daba sobre el rio a traves de Reichstagsufer. Las fotografías aereas mostraban el terreno ocupado en esos momentos por una ferla de alracciones y delataban una discrepancho con el plano respecto del puente Weidendammer. Dicho puente de uso peatonaly rodado, que debería estar a

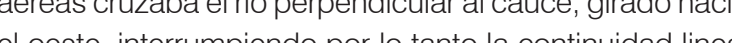
de la cale A la vista por lo tanto la conthuidad lineal como cas A la vista de las folograflas, tanto anteriores provison. La call año 1921, esta situación debía ser

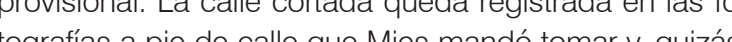
tografias a pie de calle que Mies mandó tomar y, quizás, es el molivo que orighír la serie de estudios grálicos que 


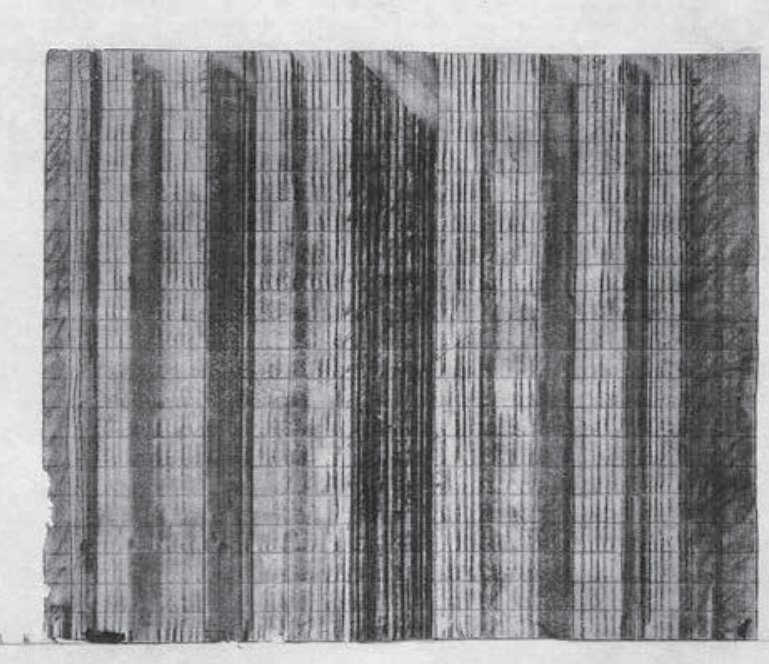

5. Azzado al río Spree. 6. A la izauierda vista hacia el norte desc vistas hacia el sur (M2).

7. Vista hacia el sur (M2). Dibuio a carbón.
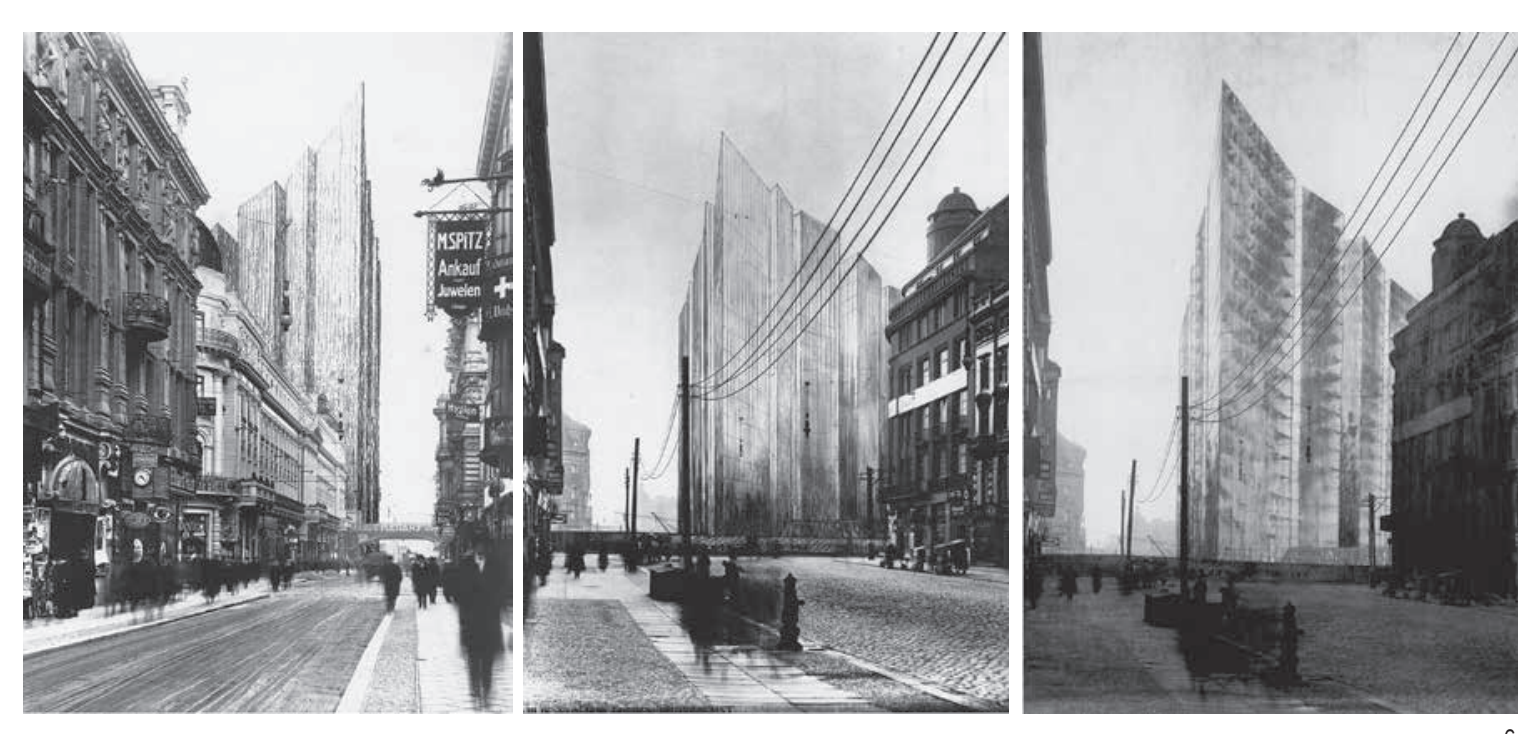

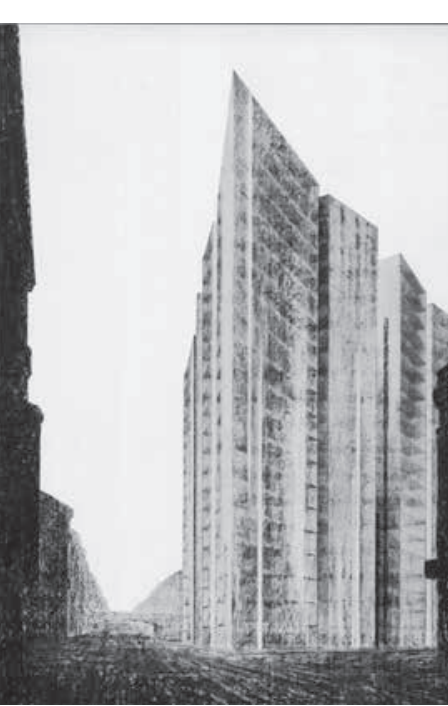

que gracias a tener una cámara de vacío en su interior posee una importante capacidad aislante"5 (figura 5).

Las vistas

Mies no se aplicó en la proyección de la planta pero si lo hizo en la preparación de las vistas. Encargó fotografías desde puntos cuidadosamente elegidos -que no coincidían con los que las bases del concurso solicitaban (punto A y punto B de la figura 4)- y dibujó la propuesta sobre grandes ampliaciones de las fotografías, muy costosas en aquel momento, eliminando de la imagen lo que no interesaba pero conservando el perfil de los edificios existentes, las farolas, los cables, la gente y los vehículos, con atención por consignar puntualmente la vida de la calle $e^{6}$. Preparó al menos tres montajes completos y un dibuio a carbón Los estudiosos coinciden en considerar los elaboraciones gráficas posteriores para publicar que pone en claro tal proceder es que Mies en lugar de preocuparse por especificar el programa -aspecto que debía considerar salvaguardado-se obstinaba en incorporar la propuesta a la ciudad.

Una de las fotografías muestra la Friedrichstrasse más o menos a la altura de la Mittelstrasse mirando hacia el norte. Desde aquí el rascacielos se alza sobrepasando largamente las vías elevadas, dominando la perspectiva de la calle, ordenando la acumulación de carteles, cornisas, anuncios, etc. El primer término aparece muy definido: la larga fachada en escorzo del Central Hotel, que ocupaba todo el frente de manzana inmediato a la estación, marca un ritmo vertical que reverbera con el trazo a carbón que representa el rascacielos. al sur o sea a contraluz La imagen desce au calle cortada por un vallado provisional antes del puente Posiblemente la interrupción de la calle sea la causa de que la actividad rodada y comercial parezca disminuida hasta casi desaparecer. Ese aspecto desolado pudo susgen, en sucesivas pruebas, que ocultan el detalle de ciudad y culminan en el dibujo a carbón de la propuesta final. Hacer un dibujo nuevo era la única manera de devoy restituir el horizonte que la fotografía, en ese momento, hurtaba La calle de este lado es más ancha la estación y las vias del tren quedan por detrás del edificio y éste se ve en toda su altura, vertical como un acantilado, dirá Franz Schulze También pueden observarse tres formas distintas de acabar el ángulo de la arista más avanzada o de traslucir el interior: marcando primero líneas verticales, estimando finalmente el sombreado de los foriados horizontales. La versión totalmente dibujada es la que difunde en las publicaciones de la época. Es la más reproducida y la que elegirá para los paneles centrales de la exposición monográfica que le dedica en el Museo de Arte Moderno de Nueva York en el año 1947 (figuras 6 y 7 ).
Las demás vistas se toman delotro lado delío a a a citar el proceso de progresivo oscurecimiento de la imaverle la continuidad a la calzada darle un suelo al edificio

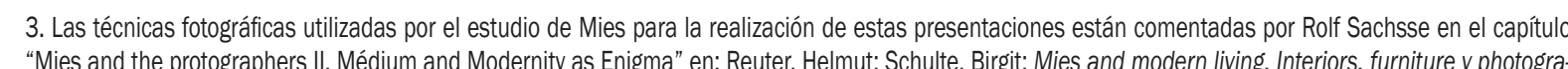
"Whes and the protographers 1 . Meddum and Moderentity
phy. Ostilidern: Hataje Canz Verlas, 2008, pp. 253-263.

4. La memoria está publicada en Frülicht, 1922 y transcrita en castellano en: Neumeyer, Fritz: Mies van der Rohe. La palabra sin artificio, reffexiones sobre arquitectura 1922/1968. Madrid: El Croquiis Editorial, 1995, pp. 362-363.

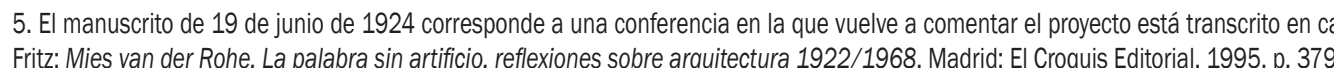
6. Las fotos de estos fotomontajes las tomó Curt Rehbein en 1922. La relación completa de los fotóórrafos que trabajaron para el estudio de Berlín de Mies hasta el año 1938 es fruto de una investigación coniunta dirigida por Helmut Reuter, Wolf Tegethoff y Rolf Sachsse publicadada en: Reuter, Helmut: Schulte, Birgitit "Mies and the protographers II. Médium and Modernity as Enigma". En Mies and modern living. Interiors, furniture and photography. Ostrildern: Hatie Canz Verlag, 2008, pp. 231-251. 

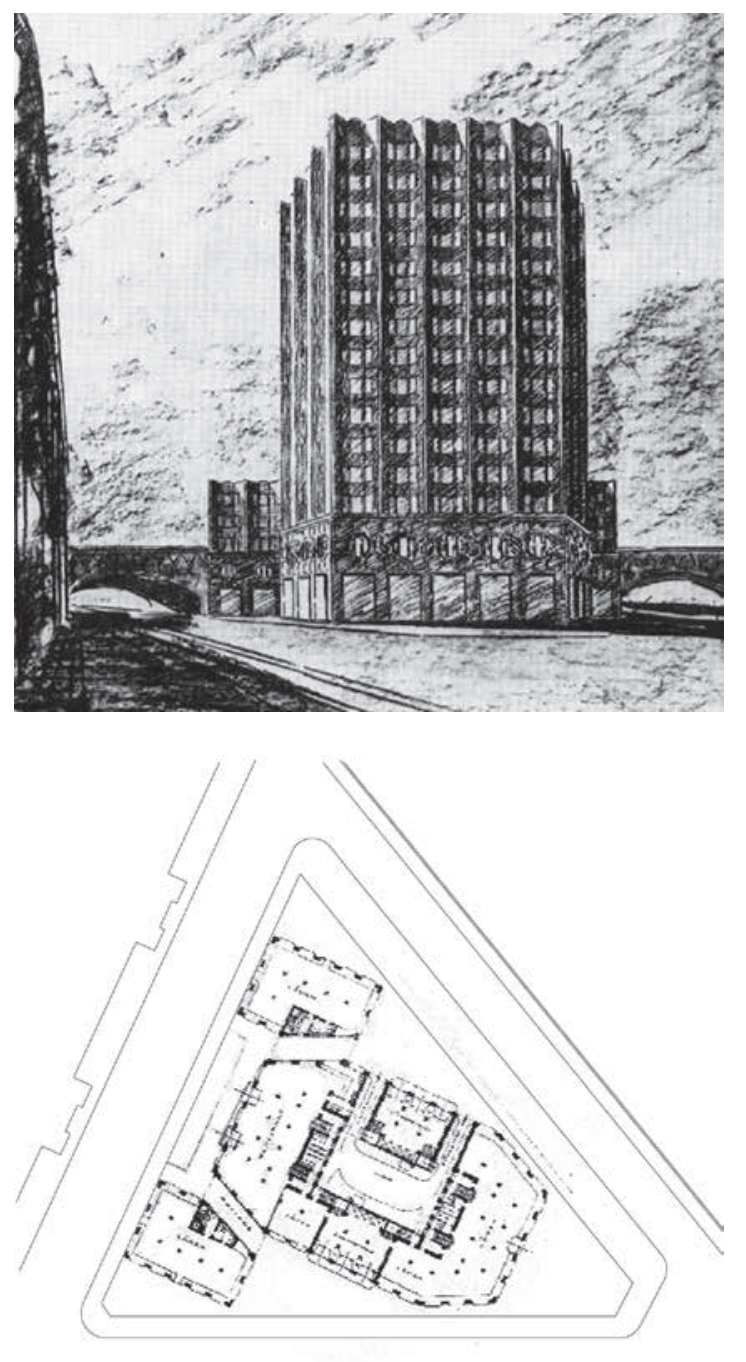

Otras propuestas

Visto el conjunto de propuestas, sorprende la variedad y el esfuerzo puesto en las presentaciones, en su gran mayoria obedientes a las bases del concurso, sobre todo en lo que se refiere a establecer una primera parte de edificio de menor altura que ocupara casi toda la parcela sobre el que elevar un cuerpo más alto. El primer premio fue para el equipo formado por Alfons Baecker, J. Brahm y R. Kasteleiner que proponia un bloque de matriz octogonal, alargado según una directriz perpendicular a la estación, al que acompananan un cuerpo bajo a cada lado que escolla los flancos del conjunto ante mada por. El segundo premio lo gano el equipo tormado por Hans y Wassili Luckhardt y Franz Hoffmann
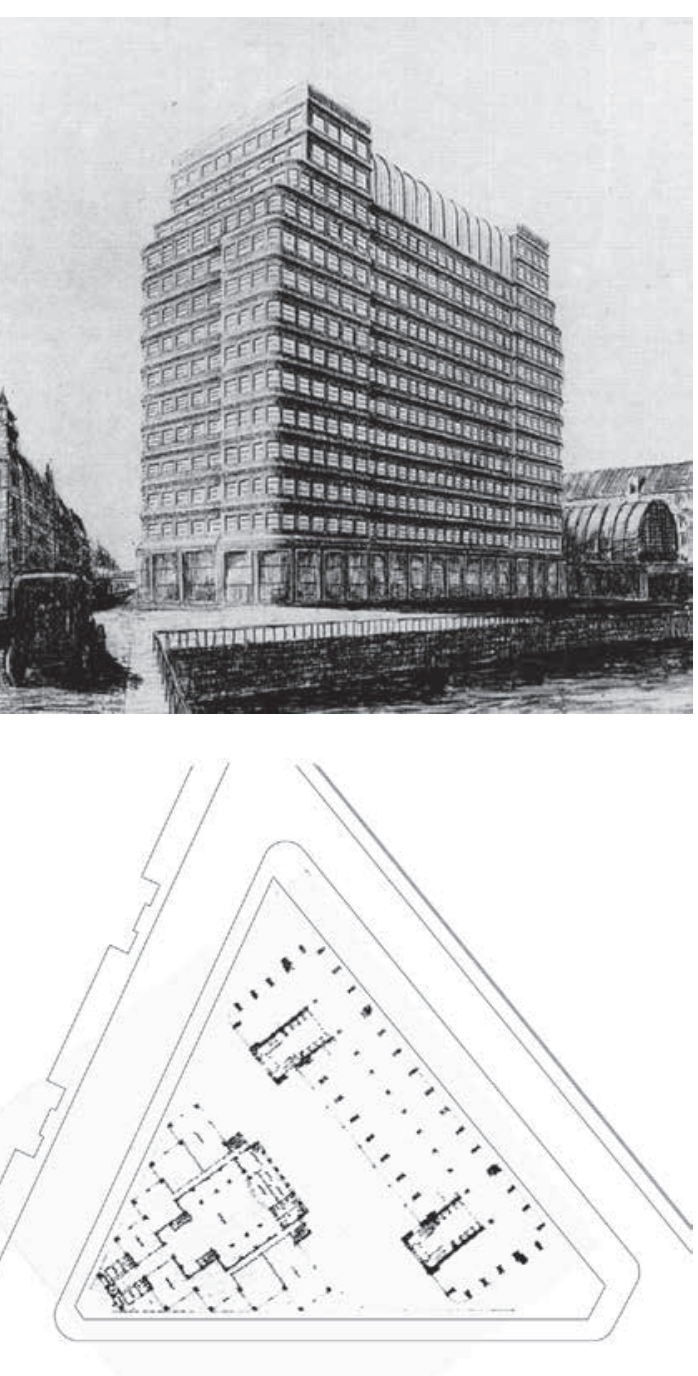

al situar el volumen principal en paralelo al río y liberar espacio delante de la estación. En resumen, ambos tomaban una u orra opción evidente respeto a las inmediaciones (figura 8 )

La propuesta de Mies suele considerarse indiferente al marco urbano por la ausencia de jerarquización aparente de los pisos, por no tener base ni coronación y por ofrecer frentes iguales en todas las fachadas?. Sin embargo esta apreciación contradice el esmero puesto en Uar a la ciudad en la representación del proyecto. Habria que apreciar en su justa medida el esfuerzo de arquitecto por localizar y seleccionar los puntos de vista Es relevante el hecho de que Mies igno los puncos de vista para las perspectivas que solicita la organización y busque unos nuevos. Necesita alejarse unas calles hacia arriba y hacia abajo para apreciar un marco de incid

Por un lado. Mies toma distancia respecto al objeto

$y$ por otro, cuida el foco y el encuadre de la mirada punto de fuga de la perspectiva estará sobre la linea del horizonte y en el ámbito de la calle, no sobre el edificio. Algo descentrado del eje para así ver la propuesta con relación a las fachadas opuestas y con los edificios que, en la misma acera, anteceden y siguen a su rascacielos. Así queda el proyecto vinculado en primer lugar a la Friedrichstrasse, el compromiso que considera más importante al ser esta calle una arteria principal de la ciudad y por ello, también con Berlín y en cierto modo, con el infinito. Es injusto considerar que el arquitecto desdeñe las circunstancias urbanas. Lo que sucede es que eleva el nivel de decisión a un rango superior, de mayor generalidad. $Y$ eso es algo que puede verse contirmado en toda su producción.

Miestodavíanolosabe perocuandotengaoportunidad de trabajar en América, lo que ahora estaba fuera de toda expectativa, pondrámáximo empeño en aligerar elencuentro del edificio con el suelo y hacerlo de un modo tal que no suponga merma del espacio público sino todo lo contrario.

1928. EN LA ESQUINA CON LA LEIPZIGERSTRASSE Unos anos mas tarde, en 1928, Mies fue invitado a participar en el concurso para la ampliación y renovación de los almacenes S. Adam. Se necesitaba ampliar y e renovar la sede actual situada en la confluencia de la Friedrichstrasse con la Leipzigerstrasse.

7. Cohen, Jean-Louis: La temptació d'Amèrica. Ciutati iarquitectura a Europa 1893-1960. Barcelona: CCCB, 1996, p. 107. 8. Las maquetas del Rascacielos de vidrio de planta curvilínea (1922) y del Edificio de oficinas de hormigón, (1923) se exhibieron juntas en la Exposición
Internacional de arquitectura de Weimar en el añn 1923 .

9. Proyecto realizado en colaboración con Paul Mahlbers, según se menciona en Reuter, Helmut; Schulte, Birgit: Mies and modern living. Interiors, furniture pholography. Ostiricm

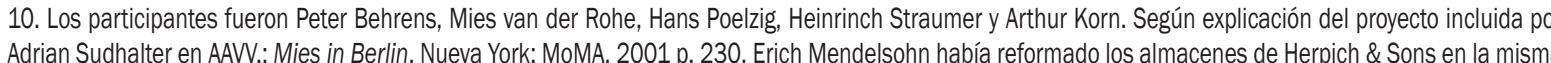
calle de Leipzigerstrasse, en el año 1925.

11. El borrador de la carta que dirige a la empresa organizadora al presentar la propuesta con la memoria justificativa es de julio de 1928 . Está transcrita en castellano en: Neumeyer, Fritz: Mies van der Rohe. La palabra sin artificio, reflexiones sobre arquitectura 1922/1968. Madrid: El Croquis Editorial, 1995, pp.
Desde 1922 Mies había desarrollado por iniciativa pros sin motivación externa conocida ni emplagran impacto por su publicación y su presentación en exposiciones: el Rascacielos de vidrio de planta curvilínea (1922), el Edificio de oficinas de hormigón (1923). la Casa de hormigon, (1923) o la Casa de ladrillo $(1924)^{8}$. Gracias con el Werkbund ha tenido ocasión de edificio de apartamentos que preside la colonia (1927). I Glass-room (1927), el café Samt \& Seide en Berlín (1927) y, en el momento de convocarse este nuevo concurso de Alemania en la Exposición Internacional de Barcelona e se celebraría el año siguiente.

政 mantener la alineación a la calle. En 1924 habia colabora(ando el edificio que ahora se ha de . explicación del proyecto es muy explícita y en la carta que ciláfanos. Además, necesitan que estos espacios sea . vista de la presentación. En este caso procura que se se vea la esquina del edfício a Leipzigerstrasse y, en escorto a la envolvente indiferenciada del rascacielos del año 列 edes neces entrega de la propuesta grafica dice: "Uszo, las dos fachadas de la calle Friedrichstrasse. Respec- 

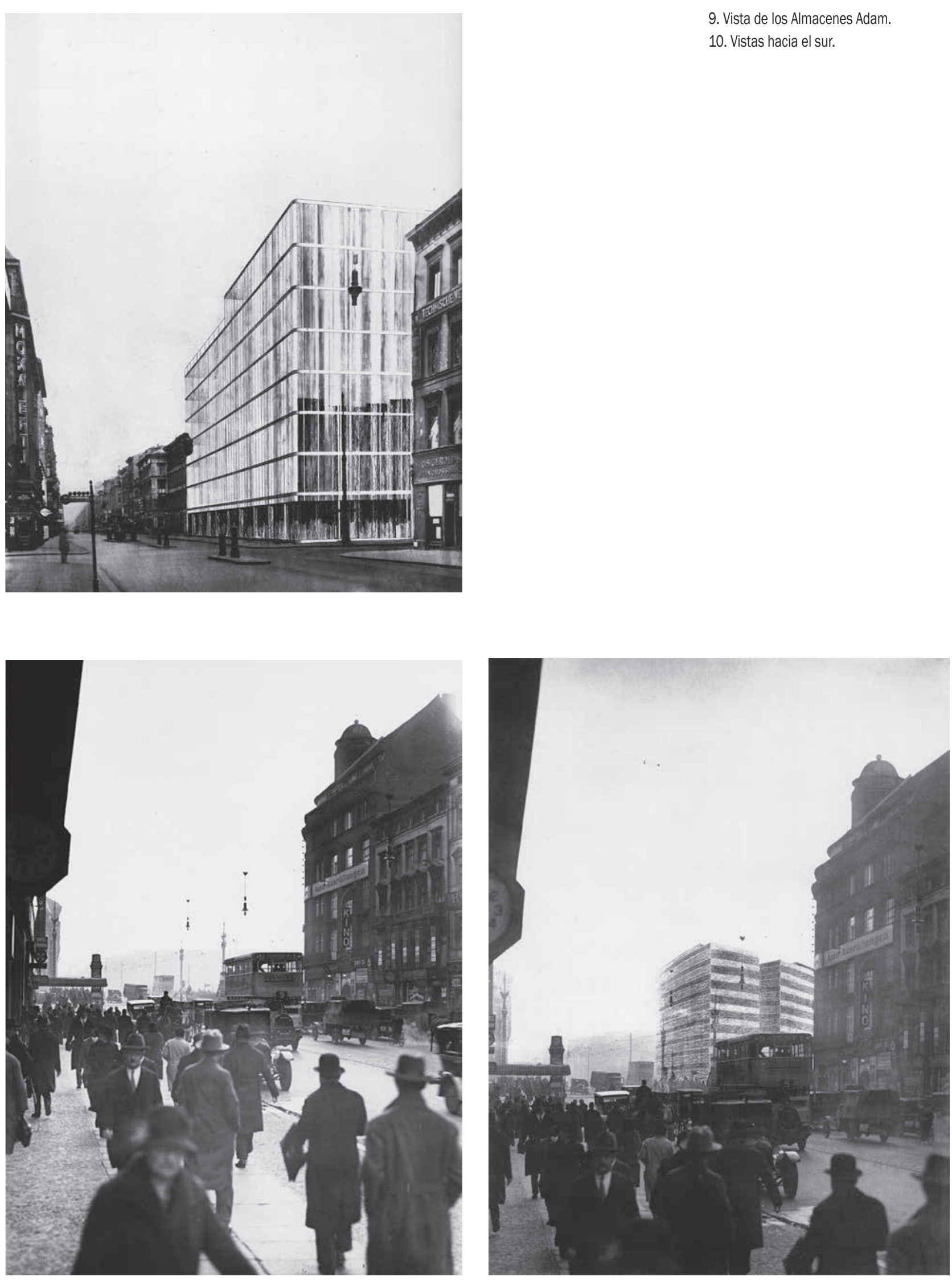

1921 destacan algunos matices. La planta baja recibe un trato diferenciado, se retrasa el cerramiento propormite detenerse en los escaparates; el plano de vidrio de fachada se descuelga un poco respecto del primer forjado lo que reduce la altura libre visible del porch y oculta el encuentro de los pilares con el foriado; la planta añadida en la zona de la esquina escalona el perfil y, el suavizado de la arista en el encuentro de los planos de fachada con un pequeño radio de curvatura, significa el empleo de lunas de vidrio curvadas para esta zona. El concurso se canceló sin dar un ganador (figura 9).

Seguidamente, en agosto de 1928, recibio la convocatoria para el proyecto de unas Oficinas y un Banco en Stuttgart, frente a la Hindenburgplatz, que le ocupara hasta diciembre y en el que obtendrá una mención honoraria. La propuesta se resolvib de manera análoga a la de los almacenes Adam. una planta baja cerrada con vidrio transparente y las superiores con vidrio traslúcido sobre el que colocar anuncios luminosos.

Tras este concurso, inicia inmediatamente el de la Remodelación de la Alexanderplatz de Berlin, ya en los primeros meses del año 1929, coincidiendo de pleno con el desarrollo del Pabelion de Alemania para la Exposición Internacional de Barcelona. La propuesta para Alexanderplatz es importante en cuanto aborda no ya un edificio sino una intervención mayor que Mies resuelve disponiendo varios edificios aislados de una forma y en un área más extensa de lo solicitado por los organizadores. Se desmarca de la tendencia en boga en aquel momento -por otra parte exigencia de las bases de concurso- de alinear la edificación con el trazado viario curvo. Queda al margen de este articulo, pero pondrá en práctica en Chicago.

1929. DE NUEVO JUNTO A LA ESTACIÓN FRIEDRICHSTRASSE

Ya avanzado 1929, a finales de septiembre, Mies recibe la propuesta de participación en el concurso para levandel rascacielos del año 1921. El nuevo promotor es la Corporación de Transporte de Berlín (Berliner Verkers-
Aktiengesellshaft). Las bases indicaban la necesidad de formalizar unos $10.000 \mathrm{~m}^{2}$ de superficie para oficinas municipales pero sin pretensiones de erigir ningún rascacielos. El área de ocupación de la parcela había disminuido un poco respecto a la del año 1921 ya que se buscaba ampliar el ancho de la Friedrichstrasse en este tramo, hasta 32 metros, y aumentar la distancia al brazo del tio Spree hasta 23 metros. Quedaban as unos $3.600 \mathrm{~m} /$ libres para ocupar. Se siguio un procedimento restringido al que se invilaron a cinco equipos: Mies van der Rohe, Afred Grenander, Erich Mendelsohn, Paul Mebes y Paul Emmerich y Heinnich Straumer de noviembre del mismo año.

La respuesta de Mies a esta segunda convocatoria es más realista e incluye estudios de distribución para verificar la planta y albergar un hotel, oficinas, establecimientos comercales o sur conexion con el subsuelo. Mies retoma el esquema de un núcleo central de acceso y servicios envuello por tres bloques rectangulares. ligeramente curvados. Con la miltad de altura que el rascacielos del año 1921, los alzados adoplan una proporción más horizontal. se pone énfasis en la alternancia de bandas de vidrio transparentes y bandas macizas, posiblemente de ladrillo rojo. Nuevamente, una solución idéntica para todas las orientaciones (figura 10). Se conceden dos primeros premios. uno a Mendesohn y otro al equipo de Mebesy Emmerich. Es sorprendente el hecho de que la volumetria de la propuesta de Mendelsohn presente rasgos en común con la premiada en segundo lugar el ano 1921: la de los hermano Luckharal. Ambos coinciden en consolidar como trente principal el lateral paralelo al iro orientando el cuerpo de mayor altura según esta dirección. Mendelsohn preparo una serie de maquelas para evaluar razonadamente hasta siete opciones diferentes y justificar la finalmente adoptada. El documento resulta de gran interes ya que las variantes que Mendelsohn analiza corresponden opciones ensayadas por los concursantes delaño 1921. Las analiza en función de la sombra que hace el edificio sobre la calle, en función de la orientación que toman los ambientes de trabajo y que repercute en su rentabilibre de parcela. Y la elegida por él de entre las que ha 

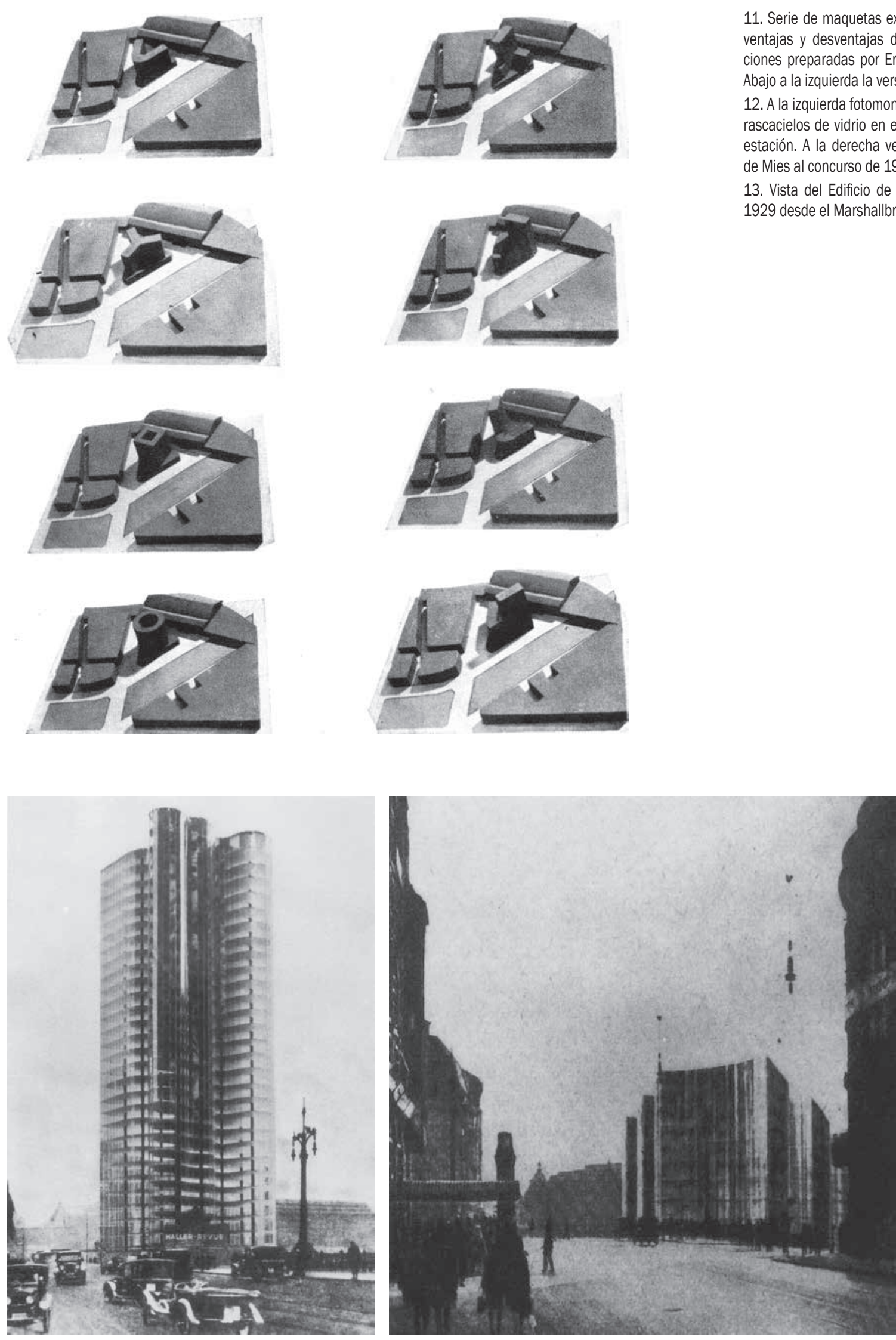

11. Serie de maquetas explicativas de las ciones preparadas por Erich Mendelsolin. Abajo a la izquierda la versión definitiva. 12. A a i izquierda fotomontaje del segundo rascacielos de vidrio en el solar junto a estación. A la derecha versión alternativa 13. Vista de Elificio de 13. Vista del Edificio de Oficinas del

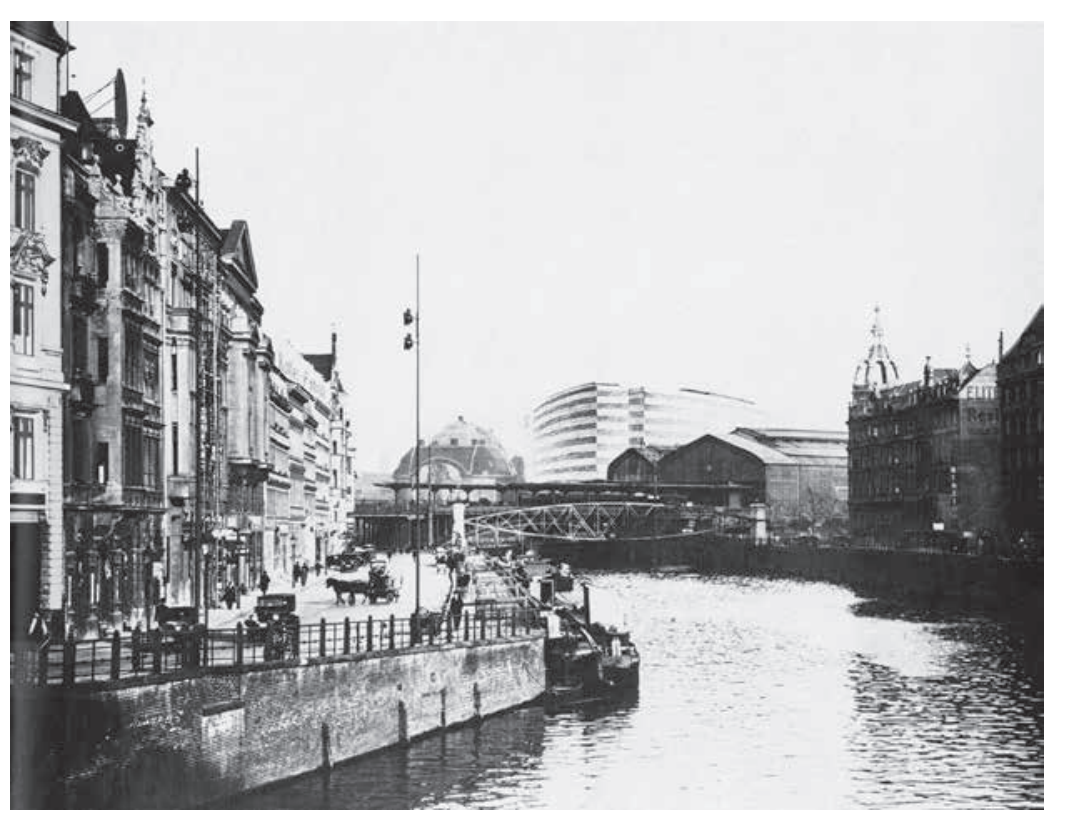
considerado, es
sitos (figura 11).

Friedrichstrasse el punto de vista de la confluencia de Izadas muestran la calle llena de actividad y tránsitua' Parece como si la ocasión le estimulara a ensayar nuevas probabilidades. Tal vez la menor altura del actual edificio de oficinas -unos 40 metros- le sugiriera contrastar el efecto que provocaría la altura del rascacielos de crista de planta curvilínea de 1922 - unos 120 metros-. Todo indica que el rascacielos de 1921 suscitó en Mies -sin mediar concurso ni encargo alguno-la propuesta para un segundo rascacielos -más alto, de 30 pisos, y perimetro sinuoso- con la intención de seguir investigando las posibilidades descubiertas en la utilización del vidrio. Hasta el momento se desconoce si existió una ubicación concreta para el rascacielos de 1922. Existe una planta delineada en un solar bien determinado por calles , pero sin nombres, y la maquela se contrastó en distintos contextos. Uno de los fotomontajes lo sitúa claramente junto al puente Weidendammer, ante la estacion Fiedrichstrasse, ensays que probablemente se hizo en este momento, varios años después de haberse proyectado con un doble propósito. medir el stio con un edificio conocido y verfiticar el edificio en distintos lugares. También se conoce una versión en la que los bloques curvilineos adoptan una configuración en arcos concentricos, aunque parece mas una prueba del despacho que una propuesta convincente (figura 12).
Mies vuelve a alejarse y encuentra un nuevo punto desde el que comprobar el volumen con la estación (punto M3 de la figura 4). Así cruzando, el río desde e puente Marshall y mirando al este, la leve convexidad de as fachadas parece afín al giro del cauce, a los arcos de la estación y al remate curvado de la Komische Oper (figura 13)

En la década de los treinta, Mies van der Rohe fue invitado a participar en los concursos para la reforma interior del Neue Wache de Schinkel -en la avenida Unter den Linden- para convertirio en un Memorial a los Cardos en la Primera Guerra Mundial (abril a julio de 1930) para el Club de goll de Krefeld (1930), la Casa Gericke (1932) on Wannsec, la anmilación del Reichsbank (1933) y por ultimo para el Pabellón Alemán en la Exposición y por uilmo pera el Pabelón Aleman en la Exposición nitivamente a Estados Unidos en 1937. Una vez alli fue Inviado al concurso para el Tearro de Manheim, en 1952 en el que se implicó personalmente. Los concursos en Estados Unidos tentan un formato muy diferente. La oficicon con sociedades promotoras pero en ellos la implicación .

EPílogo

Las impecables imágenes de las propuestas que Mies presenta a concurso dan verosimilitud a los -en aque momento- inusuales edificios acristalados. el encuadre acompaña la mirada del primer plano al infinito y les 
asigna lugar en el mundo. La distancia desde la que los contempla amplía el marco de relaciones y aumenta el rango de los vínculos que atañen a las decisiones de proyecto. Estas primeras tentativas urbanas -más allá de la squinada geometria en planta del rascacielos del año 1921 , que repercute la parcela triangular, y la del año 1922 resultado del ensayo con los reflejos del vidrio- presentan una particulandad que será definitiva. el ser de una pieza de arriba abajo. El perimetro sinuoso del rascacielos de b, sobrio y severo, al igual que su precedente angulado. Persiste a partir de entonces una clara determinación los planos de suelo horizontal se superponen identicos y ofrecen frentes de fachada equivalentes a todas las orientaciones formado una unidad elemental. Se inicia e proceso de constalación de la primacia de los aspectos de mayor generalidad, estructurales, constructivos $y$ de torma del espacio unano, en detimento del ensayo de uces y reflejos -más bien fruto de un deslumbramiento naugural. Mies persevera siempre en conducir la oporunidad de edificar a sus principios esenciales y en dar a cada solución una aplicación y significación universales. A pesar de la notoriedad que alcanzaron estos prime ros rascacielos y su papel determinante, años más tarde, en la definición del tipo en altura su propósito nunca tue el hacer el edificio lo mas alto posible, sin más. Mies, preocupado por el plano de apoyo y la percepción de la Inea del horizonte, siempre mantuvo los pies en el suelo. Se ha visto el esfuerzo por traer la calzada a las vistas de concurso. En sus posteriores intervenciones en centros urbanos -Chicago, Toronto o Montreal- Mies optará por desdoblar la superficie construida en dos o más piezas por incorporar cuerpos bajos que colaboren a delimitar e espacio peatonal.

En los años 20 la mención de la palabra rascacielos remitía inmediatamente a Nueva York. En una deliciosa crónica, Joseph Roth, novelista y cronista cultural en el
Berlín de esos años, se demoraba sólo tres frases en decir "aquellos edificios enormes que se ven en las foografilas de las calles de Nueva York". Mies, al igual que Roth, conocia esas imágenes y cuando acentúa los ángulos de fuga en las perspectivas de 1921 seguramente thlaba en su retina la imagen del edificio Flatiron - todos bs rascacielos de Nueva York en ese momento presentaban rettanqueos en altura excepto este-. En tres décadas Mies será actor principal del escenario neoyorquino. E ascacielos que erigirá en Park Avenue (1954-1958) será en adelante un referente ineludible: destacado no ya por a solución del cuerpo alto -que toca el cielo- sino por el modo en que toca el suelo.

El 12 de marzo de 1922 Both escribia para el Berliner Borsen-Counier una reseña filulada hascacielos que traschiboparcialmente, perorecomiendosulecturacompleta ${ }^{13}$ "Desde hace algunas semanas se puede ver en el Ayuntamiento una interesantisina exposicion de planos de grandes edificios. Ahora se dice que hay que acelera a construccion de un rascacielos. Será el prmer rascacielos de toda Alemania. "Rascacielos" no es un termino ecnico, sino un termino popular que designa aquellos edificios enumes que se ven en las fotograflas de las cales de Nueva York. Es un termino romántico y gráfico. Es el nombre de un edificio cuyo tejado ya ha rascado el cielo (...) Arriba, Dios ha sido sacado de su paz eterna y obligado a implicarse en nuestro minimo destino. IAh! También puede leerse que en el primer rascacielos de Berlin va a construir un gran centro de ocio. Con cines, salas de baie, licorerias, orquestas de negros, teatro de variedades y bandas de jazz. Pues la naturaleza humana no niega su debilidad ni siquiera alli donde en apariencia esta a punto de vencerla. Y si algún día lograramos consiruir un rascaplanetas y edificar en Marte, a la delegación de expertos cingenieros se sumaria una companifa colonila de cafés cantantes. Veo cómo en las nubes altas brilla un bar. Llueven los cócteles más diversos".
AAV:: Mies in Berlin. (publicado en relación con la exposición del mismo nombre). New York/Berlín: MOMA, 2001.

Conen, Jean-Louis: La temptació d'Amèrica. Ciutat i arquitectura a Europa 1893-1960. Barcelona: CCCB, 1996. Edición original: Scènes de la ve Wure Larchitecture europeenne et la tentación del'Amérique, 1893-1960. Montreal: CCA, 1995,

. Gastón, Cristina: Mies: el proyecto como revelación del lugarar. Barcelona: Fundación Caja de Arquitectos, 2005.

Lampugnani, Vittorio M.." Modernism and the Metropolis: Plans for Central berlin 1910-41" en Kleihues, J. P.; Rathberger, C. (eds): Berlin-New York .

.

Pizza, Antonio; Pla, Maurici: Viena-Berlín. Teoría, arte y arquitectura entre los siglos XIX y XX. Barcelona: Edicions UPC, 2002.

Roth, Joseph: Crónicas berlinesas. Barcelona: Minúscula, 2006.

Schulze, Franz: Mies van der Rohe una biografía critica. Madrid: Herman Blume, 1985.

Van der Rohe, Mies: The Mies van der Rohe archive. Vol. 1-22 (catálogo comentado). Nueva York: Garland, 1986-1992 (comentarios de Franz 


\section{Autor imagen y fuente bibliográfica de procedencia}

Información facilitada por los autores de los artículos: página 20, 1 (AA.W.: Concurso de Proyectos 1996. Propuestas de los equipos adjudicatarios. Sevilla: Dirección General de Arquitectura y Vivienda, Junta Andalucía, 1997, portada), 2 (Dirección General de Arquitectura y Vivienda, Junta Andalucía, tríptico); página 22, 3 (Manuel Toledo), 4 (Andrés López); página 23, 5 y 6 (Jesús Granada) 24, 7 (Fernando Alada), 8 (Jesús Granada), 9 (Fernando Alda); páginas 42, 43 y 44, 1 a 3 (El Croquis n53 0MA/Rem Koolhaas. Madrid: El Croquis, 1994, pp. 73 y 78); páginas 46, 47, 4 a 6 (AA.VV: Exposición Universal Sevilla 1992: ideas para una ordenación del recinto, Sevilla: Expo 92, Comisaría General de España, 1986); página 49, 7 (Ceccarelli, P. La construcción de la ciudad soviética. Barcelona: Gustavo Gili, S.A., 1972, figura 26, Anexo ilustraciones, p. XI), 8 (De Michelis, M.; Pasini, E. La Citta`Sovietica 1925-1937, Venecia: Marsilio Editori, 1976, p. 80); páginas 50y 51, 9 y 10 (9. De Michelis, M.; Pasini, E. La Citta 'Sovietica 1925-1937, Venecia: Marsilio Editori, 1976, pp. 81y 180); página 55,1 (Reproducido de Deutches Dokumentation fur Kunstgeschichte, Universitat Marburg, en www.fotomarburg.de); página 56, 2 (Cristina Gastón Guirao); página 57, 3 (Der Scherei nach den Turmhaus. Der IdeenwettbewerbHochhaus am Bahnhof Friedrichstrasse. Berlin 1921/22. Berlín: Argon Verlag GmbH, 1988, p. 39), 4 (Cristina Gastón Guirao en base a Der Scherei nach den Turmhaus. Der IdeenwettbewerbHochhaus am Bahnhof Friedrichstrasse. Berlin 1921/22. Berlín: Argon Verlag GmbH, 1988, p. 38); página 58, 5 (AAVV. Mies in Berlin. (publicado en relación con la exposición del mismo nombre). New York/Berlín: MOMA, 2001, p. 183); página 59, 6 (Reuter, Helmut; Schulte, Birgit. Mies and modern living. Interiors, furniture y photography. Ostfildern: Hatje Canz Verlag, 2008, p. 50, 210 y AAVV. Mies in Berlin. (publicado en relación con la exposición del mismo nombre). New York/Berlín: MOMA, 2001, p.181), 7 (AAVV. Mies in Berlin. (publicado en relación con la exposición del mismo nombre). New York/Berlín: MOMA, 2001, p. 182); página 60, 8 (Cristina Gastón Guirao en base a Der Scherei nach den Turmhaus. Der IdeenwettbewerbHochhaus am Bahnhof Friedrichstrasse. Berlin 1921/22. Berlin: Argon Verlag GmbH, 1988, pp. 45, 105); página 62, 9 y 10 (Reuter, Helmut; Schulte, Birgit. Mies and modern living. Interiors, furniture y photography. Ostfildern: Hatje Canz Verlag, 2008, pp. 230, 252, 258); página 64, 11 (Zevi, Bruno (ed): Erich Mendelsohn. Opera Completa. Turín: Testo \& Immagine, 1997, p. 197), 12 (Der Scherei nach den Turmhaus. Der IdeenwettbewerbHochhaus am Bahnhof Friedrichstrasse. Berlin 1921/22. Berlín: Argon Verlag GmbH, 1988, pp. 181, 184); página 65, 13 (Schulze, Franz. Mies van der Rohe. Una biografía crítica. Madrid: Hermann Blume, 1986 p. 155); página 69, 1 (Inv. Nr. HP 041,005. Architekturmuseum der Technischen Universität Berlin in der Universitätsbibliothek); páginas 70, 72, 73, 74, 2 a 5 (@FLC/Vegap, Sevilla, 2012. @Pierre Jeanneret, Vegap, Sevilla, 2012), 6 (Theaterwissenschaftliche Sammlung Universität zu Köln); página 75, 7 y 8 (Inv. Nr. 2750, Inv. Nr. F 1604. Architekturmuseum der Technischen Universität Berlin in der Universitätsbibliothek); páginas 76 y 80, 9 a 11 (@FLC/Vegap, Sevilla, 2012. @Alexander Rodchenko, Vegap, Sevilla, 2012), 12 (0148126. Tretyakov State Gallery, Moscow); página 82, 13 (Austrian Frederick and Lillian Kiesler Private Foundation Vienna), 14 ((СFLC/Vegap, Sevilla, 2012. @Pierre Jeanneret, Vegap, Sevilla, 2012); página 84, 15 (@FLC/Vegap, Sevilla, 2012. @Pierre Jeanneret, Vegap, Sevilla, 2012), 16 (X1119.3a-c. Colletion of Brooklyn Museum, New York); página, 85, 17 (Cabinet Ejzenstejn, Moscow (Hayuнoмемориальный кабинет-музей С. М. Эйзенштейна), 18 (Imagen de dominio público. http://commons.wikimedia.org/wiki/File:Sadanji_Ichikawa_Il_and_Sergei_Eisenstein. jpg); páginas 92 y 94, 1 y 2 (Roth, Alfred: La nouvelle architecture presentée en 20 exemples, Die neue architektur dargestellt an 20 Beispielen, The new architecture presented in 20 examples. 1930-1940. Zúrich: Verlag für Architektur Artemis, 1975, pp. 131, 132); página 94, 3 (Architettura, № XVI, Fasc. IX. Milán: septiembre de 1938, p. 574); página 96, 4 (Roth, Alfred: La nouvelle architecture presentée en 20 exemples, Die neue architektur dargestellt an 20 Beispielen, The new architecture presented in 20 examples. 1930-1940. Zúrich: Verlag für Architektur Artemis, 1975, p. 136), 5 (Fondazione Giuseppe Terragni; reproducida en Curtis, William J. R.: La arquitectura moderna desde 1900. Londres: Phaidon, 2006, p. 366); página 97, 6 (International Institute of Social History, Amsterdam; reproducida en Molema, Jan: Jan Duiker. Barcelona: Gustavo Gili, 1996, p. 85.), 7 (Emilio Cachorro Fernández); página 98, 8 y 10 (Roth, Alfred: La nouvelle architecture presentée en 20 exemples, Die neue architektur dargestellt an 20 Beispielen, The new architecture presented in 20 examples. 1930-1940. Zúrich: Verlag für Architektur Artemis, 1975, p. 137), 9 (AA.W.: Iǵnazio Gardella, 1905-1999. Arquitectura a través de un siǵlo. Catálogo de la exposición. Madrid: Ministerio de Fomento, 1999, p. 13); página 100, 11 y 12; página 102, 13 (Maffioletti, Serena: BBPR arquitectura. Banfi, Belgiojoso, Peressutti, Rogers. Sevilla: COAAO, 1996, pp. 47, 56, 67), 14 (Musée du Petit Palais, Ginebra; reproducida en Stoichita, Victor l.: Ver y no ver. Madrid: Siruela, 2005, p. 38); página 108, 1 (Arquitectura. Agosto 1969, № 128. Madrid: Colegio Oficial de Arquitectos de Madrid, 1969. Primera página; Nueva forma: arquitectura, urbanismo, diseño, ambientes, arte. "Concurso Universidad Autónoma de Madrid". Septiembre 1969. № 44. Madrid, 1969. Portada; Nueva forma: arquitectura, urbanismo, diseño, ambientes, arte. "Concurso Universidades Autónomas de Bilbao y Barcelona". Enero 1970. № 48. Madrid, 1970. Portada); página 110, 2 (Nueva forma. Septiembre 1969. № 44. cit. p. 5); página 111, 3 y 4 (Arquitectura. Agosto 1969, № 128. cit. pp. 33 y 35); páginas 112 y 113, 5 a 9 (Nueva forma. Septiembre 1969. № 44. cit. pp. 33, 30, 81, 18, 24); páginas 114, 115 y 116, 10 a 14 (Nueva forma. Enero 1970. № 48. cit. pp. 23, 20, 56, 69, 96), 15 y 16 (Cuadernos de arquitectura. Primer trimestre 1970. № 75. Barcelona: Colegio Oficial de Arquitectos de Catalunya, 1970. pp. 58 y 66); página 117, 17 (Nueva forma. Enero 1970. № 48. cit. p. 31); página 118, 18 (Nueva forma. Septiembre $1969 . N^{\circ} 44$. cit. p. 4; Temas de arquitectura y urbanismo. Septiembre 1969. № 123. Madrid-Barcelona, 1969. p. 5); página 123, 1 y 2 (Bucci, Ângelo; Puntoni, Álvaro; Vilela, José Oswaldo. Foto: Nelson Kon.); página 126, 3 (Parada, Sérgio Roberto. Projeto cedido por Rodrigo Biavati), 4 (Gorgati, Vinicius; Franco, Fernando de Mello; Moreira, Marta; Braga, Milton. Projeto cedido pelos autores), 5 (Rodrigues, Sidney Meleiros; Saraiva, Pedro Paulo de Melo; Rosemberg, Marcelo; Vaisman, Jacobina; Lobo, Marcos Toledo; Nunes, Ronaldo Soares.Projeto cedido pelos autores); páginas 128, 129, 6 a 12 (Bucci, Ângelo; Puntoni, Álvaro; Vilela, José Oswaldo. Projeto cedido pelos autores); página 131, 13 y 14 (Bucci, Ângelo; Puntoni, Álvaro; Vilela, José Oswaldo. Foto: Nelson Kon); página 136, 1 (Fromonot, Françoise. En Jørn Utzon, architetto della Sydney Opera House. Milano: Electa, Documenti di Architettura, 1998. pp. 15), 2 (Piano, Renzo. En Giornale di Bordo. Florencia: Passigli Editori, 1997, pag 38); página 138, 3 (Mikami, Yuzo. En Utzon's sphere. Sydney Opera House. How it was designed and built.Tokio: Ed. Shoku Kusha, 2001), 4 (Silver, Nathan, The making of Beuabourg, A building biography of the Centre Pomidou, Paris. Cambridge, Massachussets: Ed The MIT press, 1994); página 139, 5 (Piano, Renzo, Renzo Piano Building Workshop, complete works. Londres: Phaidon Press Limited, 1993. vol 1. pp. 54), 6 (Weston, Richard: Utzon : inspiration, vision, architecture. Hellerup (Dinamarca): Editorial Blondal, , 2002. pp. 117); página 140, 7 (Weston, Richard: Utzon: inspiration, vision, architecture. Hellerup (Dinamarca): Editorial Blondal, , 2002. pp 116), 8 (Fromonot, Françoise. En Jørn Utzon, architetto della Sydney Opera House. Milano: Electa, Documenti di Architettura, 1998. pp 64), 9 (Ferrer, Jaime, Jørn Utzon. Obras y proyectos. Barcelona: Editorial. Gustavo Gili, 2008. pp. 159); página 10, (Rice, Peter: "La structure métallique". En Architecture d'aujourd'hui. Febrero 1982, n 219. pp. 62. París: Editorial Groupe Expansion,1930), 11 (Piano, Renzo, Renzo Piano Building Workshop, complete works. Londres: Phaidon Press Limited, 1993. vol 1. pp. 45); página 142, 12 (Architectural Design', vol 47 nº 2, febrero 1977); página 144, 13 (Weston, Richard: Utzon : inspiration, vision, architecture. Hellerup (Dinamarca): Editorial Blondal, 2002); página 148, 1 (AA.W.: Arquitectura Viva. "Obras singulares". 1998, № 62. Madrid: Arquitectura Viva. 1998. pp. 78-81); página 149, 2 y 3 (AA.W.: Concurso 2G competition: Parque de la Laguna de Venecia = Venice Laggon Park. Barcelona: Gustavo Gill, 2008. pp. 81, 13); página 150, 4 (Jacopo de 'Barbari, [Web en linea]. [Consulta: 04-10-2012]), 5 (Zumthor, Peter: Atmósferas: entornos arquitectónicos, las cosas a mi alrededor. Barcelona: Gustavo Gili, 2006. p. 6); página 151, 6 (David Chipperfield 2006-2009. El Croquis. № 120. Madrid: El Croquis. 2008. p. 66); páginas 152, 153,7 a 9 (AA.W.: Carlos Ferrater. Works and Projects 1980-2000. Barcelona: Actar, 1998); páginas 154, 155, 10 a 12 (Enric Miralles + Benedetta Tagliabue: 1995-2000. El Croquis. № 72. Madrid: El Croquis. 2000. pp. 132-141); página 156, 13 a 15 (David Chipperfield 2006-2009. El Croquis. № 120. Madrid: El Croquis. 2008. pp. 64, 67, 66); página 157, 16,17 (Luca Nicolao Photography, [Web en linea]. [Consulta: 19-9-2012]), 18 (David Chipperfield 2006-2009. El Croquis. № 120. Madrid: El Croquis. 2006. p. 66); páginas 162, 164, 167, 169, 170, 1 a 6 (๑ F.L.C. /VEGAP, Sevilla, 2012. OPierre Jeanneret, VEGAP, Sevilla, 2012); página 176, 1 (García Mercadal y S. Giedion. Congresos internacionales de arquitectura moderna. AC. №5. Primer Trimestre de 1935. Barcelona. GATEPAC.1932 p.12); páginas 178, 179, 2 a 4 (AC. №11. Tercer trimestre de 1933. Barcelona. GATEPAC. 1933. pp. 37, 34, 35); página 180, 5 (Le Corbusier et Pierre Jeanneret : Ouvre Complète 1910-1929. Zurich : Les Édtions D’Architecture. 1964 p.65), 6 (AC. NN11. Tercer trimestre de 1933. Barcelona. GATEPAC. 1933. p. 34), 7 (Le Corbusier et Pierre Jeanneret: Ouvre Complète 1910-1929. Zurich : Les Édtions D’Architecture. 1964 p.65), 8 (L'Habitation minimun. Frankfurt. 1929. Zaragoza: Edición facsimil Colegio de Arquitectos de Aragón.1997.p.26), páginas 181, 182 (AC. №11. Tercer trimestre de 1933. Barcelona. GATEPAC. 1933. p. 37, 1); páginas 189, 190, 1 y 2 (Cássia de Souza Mota); página 191, 3 (Boero, Dolores; Castellote, Ana Maria; Puglisi, Jose Agustin. Fonte: http://www.uia-architectes.org/texte/ england/Celebration/SPrix/Reglll/Frames.html), 4 (Fernandez, Alberto; Guerrero, Camilo; Javiera, Paulina; Sanchez Recio, José Manuel. Fonte: http://www.celebcities2.org/); página 192, 5 y 6 (Nikita, Sergienko. Fonte: http://www.celebcities3.org/wc/content/18/pdf/054FECC2-C9DC-410A-A044-CB2A0BBD25EB.pdf) 